\title{
EFEITO DA AÇÃO COMBINADA DE FLUORETO A OUTRAS SUBSTÂNCIAS NA REMINERALIZAÇÃO DE LESÕES DE CARIE, UMA REVISÃO INTEGRATIVA.
}

\section{$\underline{\text { Carlos Sampaio de Santana Neto }}{ }^{1}$; Silvia Letícia Sena Ferreira ${ }^{2}$; Hervânia Santana da Costa $^{3}$; Ana Rita Duarte Guimarães ${ }^{4}$}

1. Bolsista/Estagiário, Departamento de Saúde, Universidade Estadual de Feira de Santana, e-mail: cs.neeto@gmail.com

2. Participante do projeto, Departamento de Saúde, Universidade Estadual de Feira de Santana, e-mail: silvialsena@gmail.com

3. Participante do projeto, Departamento de Saúde, Universidade Estadual de Feira de Santana, e-mail: hervacosta@gmail.com

4. Orientador, Departamento de Saúde, Universidade Estadual de Feira de Santana, e-mail: ardg1999@gmail.com

PALAVRAS-CHAVE: remineralização dentária; flúor; cariostáticos.

\section{INTRODUÇÃO}

A cárie dentária mostra-se como uma das afecções de maior prevalência na cavidade bucal. Atualmente, a cárie é considerada uma doença complexa, polimicrobiana, uma disbiose. A lesão de cárie é observada apenas quando há um desequilíbrio no meio favorecendo a acidificação do biofilme dentário em decorrência do metabolismo dos carboidratos da dieta por microorganismos residentes (CRUZ et al., 2017).

Lesões de cárie em estágio inicial podem ser controladas por procedimentos não-invasivos, baseados na desorganização mecânica do biofilme dental e no uso de agentes fluoretados e antimicrobianos (SÉLLOS et al., 2008). Zero (1999) afirma que os fluoretos são eficazes para interferir em tais perdas, por meio da paralisação e/ou reversão das lesões de cárie incipientes.

Em situações de alto desafio cariogênico, os níveis de $\mathrm{pH}$ ficam menores do que 4,5, e a fase de remineralização é impedida, e disponibilidade de mais fluoreto não irá, necessariamente, prover um melhor efeito cariostático. Por isso, se tornou necessário investigar outros agentes anticariogênicos que possam ter efeito sinérgico com o flúor ou que possam ser uma alternativa para aumentar a remineralização dental (ZERO, 1999). Vários métodos para potencializar o efeito cariostático do fluoreto em baixos níveis de $\mathrm{pH}$ têm sido sugeridos, dentre outros, a combinação de fluoreto com antimicrobianos (GUIMARÃES et al., 2008).

Apesar da grande quantidade de artigos publicados na Odontopediatria, pouco se sabe sobre a qualidade científica desta produção. Este estudo buscará a padronização de texto objetivo e afirmativo, identificando e classificando os desenhos de estudo de acordo com o nível de evidência científica, sobre procedimentos terapêuticos e preventivos, recomendando ou contraindicando condutas, ou ainda apontando a inexistência de informações científicas que permitam a recomendação ou a contraindicação sobre a eficácia da ação combinada de fluoreto tópico e outros agentes na remineralização de lesões de carie. 


\section{METODOLOGIA}

As bases de dados escolhidas para pesquisa foram: PubMed, Lilacs e IBICT. A partir da seleção das bases de dados, buscou-se palavras chaves que melhor de adequassem ao tema proposto por meio da consulta dos Descritores em Ciências da Saúde. As palavras chaves utilizadas para a pesquisa foram (Fluoride) AND Combined caries, (Fluoride) AND Laser e (Fluoride) AND Xylitol. Os critérios de inclusão adotados são: estudos encontrados sobre o tema em tela divulgados nos últimos dez anos, nos idiomas português, inglês e espanhol. Os trabalhos não disponíveis na íntegra e que não atenderem os critérios de inclusão foram excluídos da revisão (Figura 1).

A seleção foi feita através da leitura dos resumos, todos os estudos foram categorizados de acordo com o critério de Nível de Evidência Científica por Tipo de Estudo - "Oxford Centre for Evidence-based Medicine, 2001". As referências bibliográficas foram classificadas de ac ordo com o grau de recomendação A, B, C ou D.

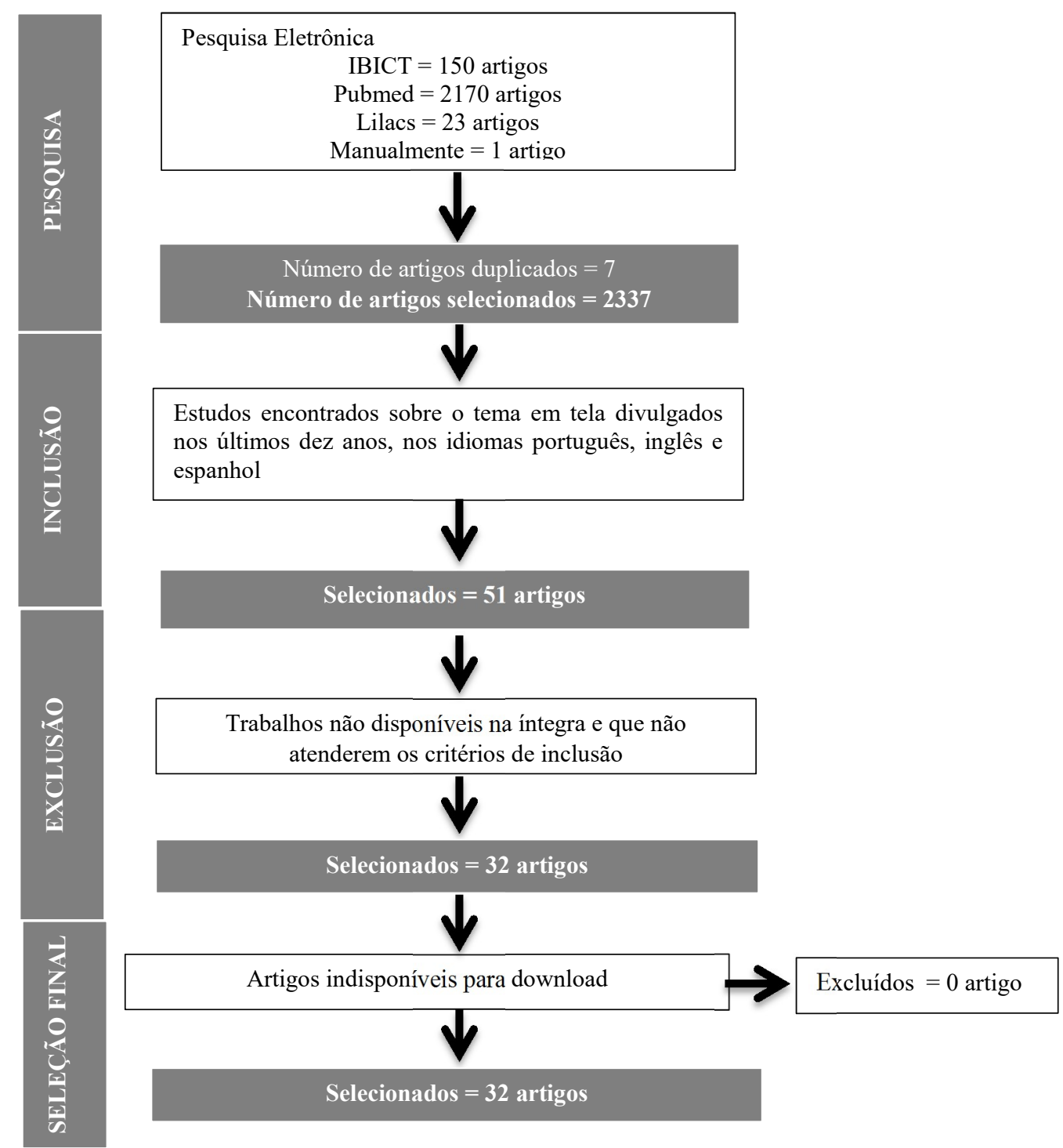

Figura 1 - Fluxograma contendo os métodos de inclusão e exclusão dos artigos. 


\section{RESULTADOS E DISCUSSÃO}

O nível de evidência por tipo de estudo, segundo a "Oxford Centre for Evidence-based Medicine, 2001", mostrou que aproximadamente 90\% (29 artigos) dos estudos tinham baixo grau de recomendação (grau de recomendação "D"), os outros 10\% (três estudos) correspondiam a estudos com grau de recomendação "A" (Tabela 1).

Tabela 1: Organização dos artigos selecionados quanto os seus autores, ano, tipo de estudo, evidência científica, materiais usados e resultado da sua utilização.

\begin{tabular}{|c|c|c|c|c|c|}
\hline Pusins & Sक्ष & Toode Exwo & $\begin{array}{l}\text { Evameas } \\
\text { Coertifras }\end{array}$ & 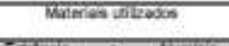 & 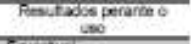 \\
\hline 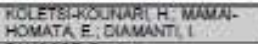 & $28=2$ & Thes & & 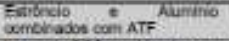 & Fanculve \\
\hline ZWUCOE, $\mathrm{a} \alpha \mathrm{WL}$ & 2006 & Tinvere & 5 & 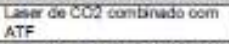 & 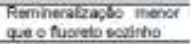 \\
\hline AEEVEDO, D. \& \& & $20 \div 2$ & inveo: & 5 & 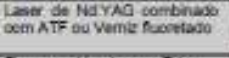 & 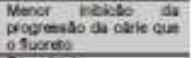 \\
\hline 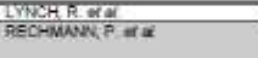 & $\frac{20 \mathrm{N1}}{2013}$ & 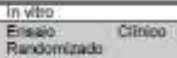 & $\frac{5}{78}$ & 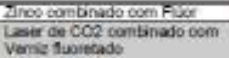 & 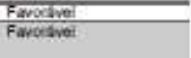 \\
\hline Du, श्या & 2072 & Tnvero & 5 & 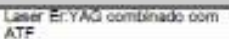 & Farobivel \\
\hline 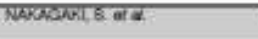 & 2075 & Tinking & 5 & 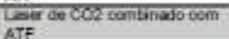 & Farober: \\
\hline ERREA 8 ; OUVER है & 2002 & Tnveros & 5 & 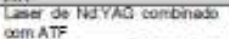 & Favartuel \\
\hline 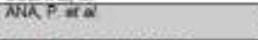 & 2015 & Tin $x$ bo: & 5 & 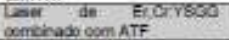 & Fanchavel \\
\hline 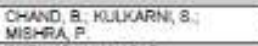 & $20+5$ & in vitro otgo & 5 & 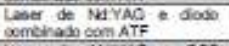 & Favorave: \\
\hline CAEK, C; HLWO 8 & 2000 & Invere & 5 & 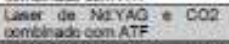 & 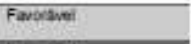 \\
\hline 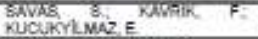 & 2016 & invertor & 5 & QTF & Faratuel \\
\hline 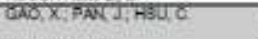 & 2006 & Tासका & 5 & $\begin{array}{l}\text { Gede de COS } \\
\text { ATf }\end{array}$ & Farorave \\
\hline BNW,$+\alpha^{\alpha} \alpha$ & 2007 & Tinvero & 5 & 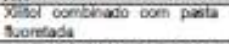 & Faworaver \\
\hline 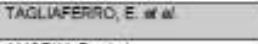 & 2008 & $\ln v_{60}$ & 5 & 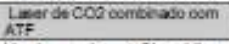 & 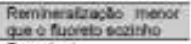 \\
\hline AMOAIM R. of al & 2007 & In vitro & 5 & 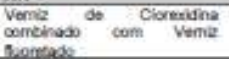 & Firotivel \\
\hline 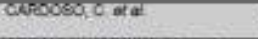 & $20 \pi 2$ & $\operatorname{Tn} \times 60$ & 5 & 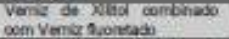 & Favonive \\
\hline OERTLDOLUNRT INS, V. QR A & 2014 & Tinverio: & 5 & 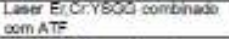 & Favorive: \\
\hline DE-ORPLI, $A$ \& ai & 2010 & $\begin{array}{l}\text { Fuso } \\
\text { Rundompase Cinneo }\end{array}$ & 18 & 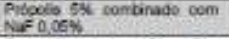 & Fancelivel \\
\hline MAATA $E \propto \alpha$ & 2013 & In vitho & 5 & 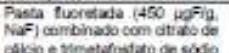 & Favalavel \\
\hline 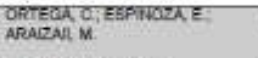 & 2073 & Thents & 5 & 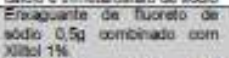 & Fancaver \\
\hline OUWWUES $A$ ब2 & 2008 & $\begin{array}{l}\text { Enivocininos } \\
\text { Randomisade }\end{array}$ & 76 & 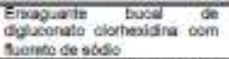 & 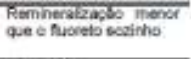 \\
\hline OLNEIRAC & 2000 & in vatso & 5 & 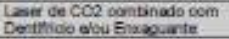 & Favasived \\
\hline QABMIEL, A & 2009 & in vitto $f$ in etse & 5 & 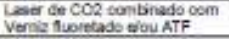 & Funodivei \\
\hline ALVEDA M & 2011 & In vea i the the & 5 & 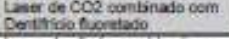 & 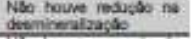 \\
\hline AEEVEDOD & 2014 & Thutro & 5 & 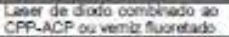 & 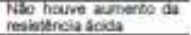 \\
\hline ZMMAATEOC & 2013 & Tines & 5 & 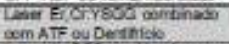 & Farosich \\
\hline VALEST, $A$ & खाना & Thvaro & 5 & 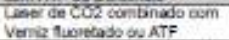 & Farstivel \\
\hline ThW $F$ & 2007 & ThW & 5 & 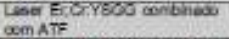 & Fanduve \\
\hline OONEB, A & 2015 & $\ln \times 60$ & 5 & 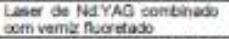 & Faveravel \\
\hline NEINA 1 & 2010 & $\ln \sqrt{60}$ & 5 & 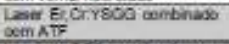 & Fawodbeil \\
\hline ZWWCOPE, & 2012 & Tn 400 & 5 & 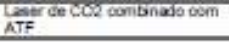 & Favortove: \\
\hline
\end{tabular}

Tanto a combinação do uso de Laser pulsos curtos quanto a combinação do Xilitol com produtos fluoretados foram os achados mais encontrados, no entanto estas duas combinações foram embasadas em estudos com baixo nível de evidência científica. Em detrimento da elevada efetividade da associação de lasers com fluoretos na remineralização das lesões de cárie, a imensa maioria dos estudos (21) tinham com grau de recomendação "D" (estudos de mais baixo nível de evidência). De forma semelhante, a despeito dos resultados favoráveis na remineralização de lesões de cárie com a combinação do xilitol com o fluoreto, apenas um dos três artigos encontrados apresentava o maior grau de recomendação (nível A).

Os resultados encontrados a partir das associações foram categorizados em "favoráveis", quando estes apresentavam um efeito significativamente positivo da ação conjunta dos 
elementos em relação ao uso separado, como: formação de $\mathrm{CaF}_{2}$ ligado a superfície do esmalte, inibição da progressão de cárie e remineralização das amostras. Levando em conta essa classificação, aproximadamente 81\% (26 artigos) dos estudos encontrados apresentaram resultados favoráveis. Devemos destacar a associação dos produtos fluoretados aos Lasers de pulso curto, ao Própolis de Apis melífera e ao Xilitol.

Houve uma diversidade de veículos dos produtos fluoretado utilizados (dentifrícios, enxaguatorios, géis e vernizes) e por conseguinte, diferenças na forma de aplicação, tempo de utilização e características inerentes a cada veículo encontrado nesta pesquisa. Desta forma, a comparação dos resultados encontrados quanto à efetividade de cada veículo fluoretado fícou comprometida.

Uma grande quantidade de estudos utilizou a associação de laser de pulso curto ao flúor e a comparação dos resultados da paralisação de lesões cariosas também ficou comprometida. A diferença na metodologia dos estudos, na análise dos dados obtidos e das diferentes configurações de laser contribuiu para isso. A irradiação com laser de $\mathrm{CO}_{2}$ foi a mais utilizada e obteve muitos resultados favoráveis em vários estudos, sendo que a combinação deste lazer com o flúor pode ser considerada a mais promissora.

Os resultados demonstraram que apesar da grande presença de artigos nas bases de dados sobre este assunto, não há grande evidência científica neles. Por isso, não é indicado o uso dessas técnicas em atividades clínicas, visto que seus efeitos e resultados ainda devem ser estudados mais afundo. Todos os materiais apresentados neste artigo devem ser analisados em mais pesquisas contendo desta vez desenhos de estudo que possuam maior evidência científica.

\section{CONCLUSÃO}

Conclui-se que, devido ao pequeno número de artigos com alta evidência científica há uma inexistência de informações científicas suficientes que permitam analisar a eficácia da ação combinada do fluoreto tópico combinado a outros agentes na remineralização de lesões de cárie, não sendo possível recomendar ou a contraindicar seu uso.

\section{REFERÊNCIAS}

CRUZ, L. R. et al. "Cárie é transmissível?" Tipo de informação sobre transmissão da cárie em crianças encontrada através da ferramenta de busca Google ${ }^{\circledR}$. Rev. Bras. Odontol., Rio de Janeiro, v.74, n.1, p.70-3, 2017.

SÉLLOS, M. C. et al. Avaliação clínica do efeito adicional do verniz fluoretado sobre a inativação de lesões iniciais de cárie em dentes decíduos. Cienc. Odontol. Bras., Rio de Janeiro, v.11, n.3, p.66-73, 2008.

ZERO, D. T. Dental caries process. Dent Clin North Am, v.43, n.4, p.635-64, Oct, 1999.

GUIMARÃES, A. R. et al. Effectiveness of two mouth rinses solutions in arresting caries lesions: a short-term clinical trial. Oral Health Prev Dent, v.6, n.3, p.231-239, 2008. 